OPEN ACCESS

Edited by:

Dirk Tischler,

Freiberg University of Mining

and Technology, Germany

Reviewed by:

Toru Matsui,

University of the Ryukyus, Japan

Pablo Ivan Nikel,

The Novo Nordisk Foundation Center for Biosustainability (DTU Biosustain),

Denmark

${ }^{*}$ Correspondence:

Nobuya Itoh

nbito@pu-toyama.ac.jp; itohnobuya123@gmail.com

Specialty section:

This article was submitted to Microbial Physiology and Metabolism,

a section of the journa

Frontiers in Microbiology

Received: 02 September 2017 Accepted: 09 November 2017

Published: 27 November 2017

Citation:

Toda H and ltoh N (2017)

Development of a Novel Escherichia coli-Kocuria Shuttle Vector Using the Cryptic pKPAL3 Plasmid from K. palustris IPUFS-1 and Its Utilization in Producing Enantiopure (S)-Styrene Oxide. Front. Microbiol. 8:2313 doi: 10.3389/fmicb.2017.02313

\section{Development of a Novel Escherichia coli-Kocuria Shuttle Vector Using the Cryptic pKPAL3 Plasmid from $K$. palustris IPUFS-1 and Its Utilization in Producing Enantiopure (S)-Styrene Oxide}

\author{
Hiroshi Toda and Nobuya Itoh* \\ Department of Biotechnology, Biotechnology Research Center, Toyama Prefectural University, Imizu, Japan
}

The novel cryptic pKPAL3 plasmid was isolated from the Gram-positive microorganism Kocuria palustris IPUFS-1 and characterized in detail. PKPAL3 is a circular plasmid that is 4,443 bp in length. Open reading frame (ORF) and homology search analyses indicated that PKPAL3 possesses four ORFs; however, there were no replication protein coding genes predicted in the plasmid. Instead, there were two nucleotide sequence regions that showed significant identities with untranslated regions of $K$. rhizophila DC2201 (NBRC 103217) genomic sequences, and these sequences were essential for autonomous replication of PKPAL3 in Kocuria cells. Based on these findings, we constructed the novel Escherichia coli-Kocuria shuttle vectors pKITE301 (kanamycin resistant) and pKITE303 (thiostrepton resistant) from pKPAL3. The copy numbers of the constructed shuttle vectors were estimated to be 20 per cell, and they exhibited low segregation stability in Kocuria transformant cells in the absence of antibiotics. Moreover, constructed vectors showed compatibility with the other $K$. rhizophila shuttle vector pKITE103. We successfully expressed multiple heterologous genes, including the styrene monooxygenase gene from Rhodococcus sp. ST-10 (rhsmo) and alcohol dehydrogenase gene from Leifsonia sp. S749 (Isadh), in K. rhizophila DC2201 using the pKITE301P and pKITE103P vectors under the control of the glyceraldehyde 3-phosphate dehydrogenase (gapdh) promotor. The RhSMO-LSADH co-expressing $K$. rhizophila was used as a biocatalyst in an organic solvent-water biphasic reaction system to efficiently convert styrene into $(S)$-styrene oxide with $99 \%$ ee in the presence of 2-propanol as a hydrogen donor. The product concentration of the reaction in the organic solvent reached $235 \mathrm{mM}$ after $30 \mathrm{~h}$ under optimum conditions. Thus, we demonstrated that this novel shuttle vector is useful for developing biocatalysts based on organic solvent-tolerant Kocuria cells.

Keywords: Kocuria rhizophila, Kocuria palustris, styrene monooxygenase, styrene oxide, biphasic reaction system, organic solvent-tolerant biocatalyst 


\section{INTRODUCTION}

Enantiopure epoxides are useful building blocks for synthesizing various chiral materials, including pharmaceuticals, agrochemicals, and fine chemicals (Farina et al., 2006; Patel, 2008). Direct enantioselective epoxidation of prochiral alkenes is a straightforward strategy for producing chiral epoxides, and many chemical approaches have been developed to achieve this objective (Shi, 2004). Sharpless epoxidation is an efficient procedure for oxidizing allylic alcohols using a titanium/tartrate/tert-butyl hydroperoxide system to yield corresponding chiral epoxides. Many chemical catalysts that contain transition metals, such as metal-salen complexes and chiral metalloporphyrins, have been developed for the enantioselective epoxidation of alkenes (Katsuki and Sharpless, 1980; Hanson and Sharpless, 1986; Irie et al., 1991; Chang et al., 1994; Tu et al., 1996). Furthermore, the biological synthesis of epoxides using monooxygenases has also been studied (Panke et al., 1998; Martinez and Stewart, 2000; Hollmann et al., 2003; Farinas et al., 2004; Champreda et al., 2006). Biological synthesis of epoxides has several advantages over chemical synthesis, including superior chemoselectivity, regioselectivity, and enantioselectivity, as well as improved environmental sustainability.

The genomes of several styrene-degrading microorganisms contain styrene monooxygenase (SMO) genes, which are involved in the first step of styrene degradation and allow them to catalyze the epoxidation of styrene to enantiopure (S)-styrene oxide (Hartmans et al., 1990; Beltrametti et al., 1997; Panke et al., 1998). SMOs consist of two enzymes: flavin adenine dinucleotide (FAD)-dependent monooxygenase (StyA) and $\mathrm{NAD}(\mathrm{H})$-dependent flavin oxidoreductase (StyB). SMOs convert styrene to $(S)$-styrene oxide with high enantioselectivity using NADH as an electron donor (Panke et al., 1999; Hollmann et al., 2003). Many SMOs are well characterized, and their enzymatic properties and substrate specificities have been investigated (Marconi et al., 1996; Panke et al., 1998; Velasco et al., 1998; Lin et al., 2010; Tischler et al., 2012). We have also reported the isolation and characterization of SMO genes from Rhodococcus sp. ST-10 (RhSMO; Toda and Itoh, 2012; Toda et al., 2012a) and the development of biocatalysis reactions for producing enantiopure epoxides from various aryl- and aliphatic alkenes using RhSMO (Toda et al., 2012b, 2014).

Escherichia coli is the microorganism most frequently used as biocatalyst host cells owing to its ease of use and abundant molecular tools for genetic manipulation. However, E. coli cells are unsuitable for long-term biocatalysis reactions using organic solvents and toxic compounds because they are easily inactivated by these substances. To overcome this problem, organic solvent-tolerant microorganisms, especially Pseudomonas putida, have been assessed as host cells for biocatalysis (Heipieper et al., 2007; Park et al., 2007; Verhoef et al., 2009; Siriphongphaew et al., 2012; Nikel et al., 2014). Recently, we also reported the construction of a biocatalysis system possessing RhSMO that uses the organic solvent-tolerant microorganism K. rhizophila DC2201 as a host cell and demonstrated the bioproduction of various enantiopure (S)-epoxyalkanes in an organic solvent-water biphasic reaction (Toda et al., 2015). K. rhizophila DC2201 is a Gram-positive microorganism belonging to the family Micrococcaceae in the order Actinomycetales, and its whole genomic DNA sequence was previously determined (Takarada et al., 2008). This microorganism has several advantages for utilization as a biocatalysis host cell, including its organic solvent tolerance, high halotolerance, robust cell structure, and small genome size. Furthermore, it was reported that several Kocuria species produce important natural pigments, including astaxanthin and $\beta$-carotene, which are widely used as food additives and health supplements (Goodwin, 1980; Porter and Spurgeon, 1981; Dufosse, 2006). It is expected that development of genetic modification tools for these bacteria may contribute to the efficient production of various useful materials such as pharmaceutical and functional food components.

Thus, Kocuria species are expected to be useful host cells for biocatalysis. However, only a few genetic tools are available for Kocuria cells (Matsumura et al., 2012; Ohta et al., 2014) and little is known about gene transcription, protein expression, and metabolite flux in Kocuria cells. Such tools and information are important for increasing the potential uses of host cells by controlling recombinant protein expression and modification of the metabolite pathways of host cells. Therefore, we aimed to both construct genetic tools for use in the genus Kocuria and apply them to a biocatalysis system for producing a wide variety of organic compounds.

In our previous study, we reported the isolation and characterization of the two cryptic plasmids pKPAL1 and PKPAL2 from $K$. palustris IPUFS-1 and the construction of the E. coli-Kocuria shuttle vector pKITE101 series based on pKPAL1 (Toda et al., 2017). These plasmids were inferred to be theta-replicating plasmids with copy numbers of 60 per chromosome in Kocuria cells. The constructed shuttle vector was stably maintained in K. rhizophila DC2201 cells, and it was available for heterologous gene expression under control of the gapdh promoter.

In this study, we isolated and characterized pKPAL3, another novel cryptic plasmid from K. palustris IPUFS-1. The whole nucleotide sequence of pKPAL3 was determined, and four putative open reading frames (ORFs) and two regions homologous to K. rhizophila DC2201 genomic DNA sequences were confirmed. The copy numbers of E. coli-Kocuria shuttle vectors pKITE301 and pKITE303 were assayed, and their segregation stability and compatibility with other E. coli-Kocuria shuttle vectors were examined. Moreover, we constructed a biocatalyst co-expressing RhSMO and LSADH (alcohol dehydrogenase from Leifsonia sp. S749) using pKITE301 and pKITE103, and the bioproduction of enantiopure (S)-styrene oxide from styrene in an organic solvent-water biphasic reaction system using 2-propanol as a hydrogen donor was demonstrated. 


\section{MATERIALS AND METHODS}

\section{Chemicals}

Styrene and styrene oxide were purchased from Nacalai Tesque, Inc. (Kyoto, Japan). Bis-(2-ethylhexyl) phthalate (DEHP) and other chemicals were purchased from Wako Pure Chemical Industries (Osaka, Japan).

\section{Culture Strains and Vectors}

Kocuria palustris IPUFS-1 (Toda et al., 2017) was used as a source of cryptic plasmids. K. rhizophila DC2201 (NBRC 103217), K. kristinae NBRC 15354, K. varians NBRC15358, K. palustris NBRC 16318, K. rhizophila NBRC 16319, K. polaris NBRC 103063, K. flava HO-9041 (NBRC 107626), and K. turfanensis HO-9042 (NBRC 107627) were used as transformation hosts for the constructed shuttle vectors, while E. coli JM109 and EC100D pir-116 were used for cloning. pHSG298 and pUC118 were used to construct the E. coli-Kocuria shuttle vectors. pGEM-T Easy Vector (Promega Corp., Fitchburg, WI, United States) was used to construct control plasmids for quantitative polymerase chain reaction (qPCR). The bacterial strains and plasmids used in this study are listed in Table $\mathbf{1 .}$

\section{Isolation and Characterization of pKPAL3 from $K$. palustris IPUFS-1}

Standard techniques were used for DNA manipulation (Sambrook and Russell, 2001). Cryptic plasmids were extracted from $K$. palustris IPUFS-1 cells using a previously described method (Toda et al., 2017). To obtain pure plasmid samples, extracted plasmids were purified by $\mathrm{CsCl}$ density-gradient ultracentrifugation and separated by agarose gel electrophoresis. Plasmids were then extracted from agarose gel and purified using the Wizard SV Gel and PCR Clean-Up System (Promega Corp.). Transposons were inserted into the purified plasmid with the EZ-Tn5TM $<$ R6Kyori/KAN-2: kanamycin resistant $>$ Insertion Kit (AR Brown, Tokyo, Japan) according to the manufacture's protocol. Plasmids with transposons inserted were introduced into E. coli EC100D pir-116 competent cells, and transformed cells were selected on LB agar plates containing $100 \mu \mathrm{g} / \mathrm{mL}$ kanamycin. Selected transformants were cultured in $4 \mathrm{~mL}$ of

TABLE 1 | Bacterial strains and plasmids in this study.

\begin{tabular}{|c|c|c|}
\hline Bacterial strain/Plasmid & Description & Source \\
\hline \multicolumn{3}{|l|}{ Bacterial strain } \\
\hline Escherichia coli JM109 & 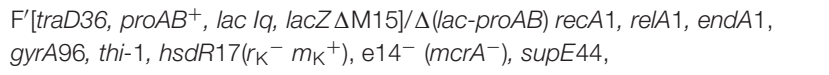 & TaKaRa \\
\hline E. coli EC100D pir-116 & $\begin{array}{l}\mathrm{F}^{-} \text {mcrA } \Delta(\text { mrr-hsdRMS-mcrBC) } \Delta 80 \mathrm{~d} / a c Z \Delta \mathrm{M} 15 \Delta / \mathrm{acX} 74 \text { recA1 endA1 } \\
\operatorname{araD139} \Delta(\mathrm{ara}, \text { leu }) 7697 \text { gall galK } \lambda \text {-rpsL nupG pir-116(DHFR) }\end{array}$ & AR Brown \\
\hline Kocuria palustris IPUFS-1 & Wild-type & Toda et al., 2017 \\
\hline K. rhizophila DC2201 & Wild-type & NBRC \\
\hline \multicolumn{3}{|l|}{ Plasmid } \\
\hline pHSG298 & E. coli cloning vector & \\
\hline pUC118 & E. coli cloning vector & \\
\hline pKPAL3 & 4.4 kbp wild-type plasmid from K. palustris IPUFS-1 & This study \\
\hline pKES3300 & E. coli-Kocuria shuttle vector harboring $4.4 \mathrm{kbp}$ fragment from pKPAL3; $\mathrm{Km}^{\mathrm{r}}$ & This study \\
\hline pKES3301 & E. coli-Kocuria shuttle vector harboring $3.4 \mathrm{kbp}$ fragment from pKPAL3; $\mathrm{Km}^{\mathrm{r}}$ & This study \\
\hline pKES3302 & E. coli-Kocuria shuttle vector harboring $2.4 \mathrm{kbp}$ fragment from pKPAL3; $\mathrm{Km}^{\mathrm{r}}$ & This study \\
\hline pKES3303 & E. coli-Kocuria shuttle vector harboring $1.4 \mathrm{kbp}$ fragment from pKPAL3; $\mathrm{Km}^{\mathrm{r}}$ & This study \\
\hline pKES3305 & E. coli-Kocuria shuttle vector harboring $3.4 \mathrm{kbp}$ fragment from pKPAL3; Km & This study \\
\hline pKES3306 & E. coli-Kocuria shuttle vector harboring $2.5 \mathrm{kbp}$ fragment from pKPAL3; $\mathrm{Km}^{\mathrm{r}}$ & This study \\
\hline pKES3307 & E. coli-Kocuria shuttle vector harboring $1.5 \mathrm{kbp}$ fragment from pKPAL3; $\mathrm{Km}^{\mathrm{r}}$ & This study \\
\hline pKES3309 & E. coli-Kocuria shuttle vector harboring $1.4 \mathrm{kbp}$ fragment from pKPAL3; $\mathrm{Km}^{\mathrm{r}}$ & This study \\
\hline pKES3310 & E. coli-Kocuria shuttle vector harboring $1.0 \mathrm{kbp}$ fragment from pKPAL3; Km & This study \\
\hline pKES3311 & E. coli-Kocuria shuttle vector harboring $0.6 \mathrm{kbp}$ fragment from pKPAL3; $\mathrm{Km}^{\mathrm{r}}$ & This study \\
\hline pKES3312 & E. coli-Kocuria shuttle vector harboring $0.8 \mathrm{kbp}$ fragment from pKPAL3; $\mathrm{Km}^{\mathrm{r}}$ & This study \\
\hline pKES3300 $\Delta$ orf1 & E. coli-Kocuria shuttle vector harboring $3.7 \mathrm{kbp}$ fragment from pKPAL3; $\mathrm{Km}^{\mathrm{r}}$ & This study \\
\hline pKES3300 $\Delta$ orf2 & E. coli-Kocuria shuttle vector harboring $3.4 \mathrm{kbp}$ fragment from pKPAL3; $\mathrm{Km}^{\mathrm{r}}$ & This study \\
\hline pKES3300 $\Delta$ orf12 & E. coli-Kocuria shuttle vector harboring $2.4 \mathrm{kbp}$ fragment from pKPAL3; $\mathrm{Km}^{\mathrm{r}}$ & This study \\
\hline pKES3300 $\Delta$ orf3 & E. coli-Kocuria shuttle vector harboring $4.0 \mathrm{kbp}$ fragment from pKPAL3; $\mathrm{Km}^{r}$ & This study \\
\hline pKES3300 $\Delta$ orf4 & E. coli-Kocuria shuttle vector harboring $3.5 \mathrm{kbp}$ fragment from pKPAL3; $\mathrm{Km}^{\mathrm{r}}$ & This study \\
\hline pKITE301 & E. coli-Kocuria shuttle vector harboring $1.0 \mathrm{kbp}$ fragment from pKPAL3; $\mathrm{Km}^{\mathrm{r}}$ & This study \\
\hline pKITE301P & $\begin{array}{l}\text { Lac promoter of pKITE301 is replaced by gapdh promoter of } K \text {. rhizophila } \\
\text { DC2201 }\end{array}$ & This study \\
\hline pKITE303 & E. coli-Kocuria shuttle vector harboring $1.0 \mathrm{kbp}$ fragment from pKPAL3; Thior & This study \\
\hline pKITE103P-LSADH & LSADH expression vector & Toda et al., 2017 \\
\hline
\end{tabular}


LB liquid medium containing $100 \mu \mathrm{g} / \mathrm{mL}$ kanamycin, and the plasmids were prepared using the Wizard Plus SV Minipreps DNA purification system (Promega Corp.). DNA sequencing was conducted using a 3130 capillary DNA sequencer (Applied Biosystems, Foster City, CA, United States) to determine the nucleotide sequences of the obtained plasmid. The primers used for the determination of the nucleotide sequences are shown in Supplementary Table S1.

\section{Construction of E. coli-Kocuria Shuttle Vectors}

To determine the minimum region of pKPAL3 required for autonomous replication in Kocuria cells, deletion clones were derived from pKPAL3 (Figure 1). Various pKPAL3 DNA fragments were obtained by PCR using the primers listed in Supplementary Table S1. The amplified DNA fragments were treated with the restriction endonucleases BamHI and SalI and ligated with pHSG298 that had been amplified by PCR and incubated with BglII and SalI. Ligated plasmids were then transformed into E. coli JM109 and selected on LB agar plates containing $100 \mu \mathrm{g} / \mathrm{mL}$ kanamycin. K. rhizophila DC2201 cells were transformed with pKPAL3 derivatives (pKES3300 to pKES3312 and pKES3300 $\Delta$ orf1 to pKES3300 $\Delta$ orf4) according to methods detailed in our previous report (Toda et al., 2017), and transformants were selected on SOB agar $(2 \%[w / v]$ tryptone, $0.5 \%[w / v]$ yeast extract, $10 \mathrm{mM} \mathrm{NaCl}, 2.5 \mathrm{mM} \mathrm{KCl}$, $10 \mathrm{mM} \mathrm{MgSO}_{4}, 10 \mathrm{mM} \mathrm{MgCl}_{2}, \mathrm{pH} 7.0$ ) containing $400 \mu \mathrm{g} / \mathrm{mL}$ kanamycin.

To construct the pKITE301 vector (Figure 2), the PCRamplified fragment of pKPAL3 (amplified using primers pKPAL3Fdel5nco and pKPAL3Rdel2bgl) was incubated with the restriction endonucleases $\mathrm{NcoI}$ and $\mathrm{Bg} / \mathrm{II}$ and ligated with a portion of pHSG298 that was PCR amplified using primers pHSG298Fbgl and pHSG298Rnco and incubated with the same restriction endonucleases. The ligated plasmid was transformed into E. coli JM109 to yield pKITE301. Similarly, the PCR fragment of pKPAL3 described above was ligated with a portion of pUC118 PCR amplified using primer pHSG298Fbgl and pHSG298Rnco and incubated with NcoI and BglII, and it was then transformed into E. coli JM109. The obtained plasmid was cut with BglII and ligated with the thiostrepton resistance gene $(t s r)$ amplified by PCR to yield pKITE303. All transformation experiments were performed three times, and transformation efficiencies were expressed as means and standard deviations (SDs).

Plasmid copy number was estimated using real-time qPCR, and plasmid stability in Kocuria cells was determined according to the methods described in a previous study (Toda et al., 2017). The primers used for estimating copy number by real-time qPCR are shown in Supplementary Table S1.

\section{Transformation of Various Kocuria Species}

Competent Kocuria spp. cells were prepared by washing cells with glycerol solution as described previously (Toda et al., 2017). Each microorganism was grown on LB medium

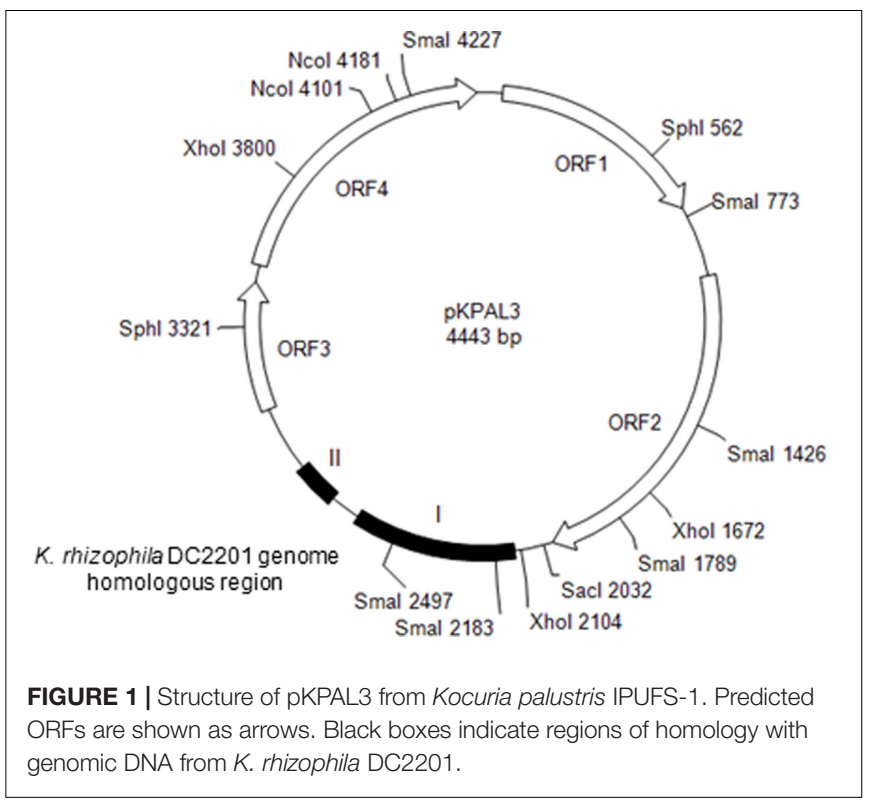

supplemented with $1.5 \%(w / v)$ glycine, except $K$. polaris NBRC 103063 , to an $\mathrm{OD}_{660}$ of 0.7 at $30^{\circ} \mathrm{C}$ with shaking. The harvested cells were washed twice with sterilized water and resuspended in $10 \%(v / v)$ glycerol with $\mathrm{OD}_{660}$ adjusted to 50. Electroporation was conducted with $0.5 \mu \mathrm{g}$ of the pKITE301 plasmid, and cells were cultured on a selection plate (SOB medium containing 100 or $200 \mu \mathrm{g} / \mathrm{mL}$ kanamycin). All transformation experiments using Kocuria spp. were performed three times, and transformation efficiencies were expressed as means and SDs.

\section{Development of a RhSMO and LSADH Co-expressing Biocatalyst}

To develop a K. rhizophila DC2201 biocatalyst that expresses both RhSMO and LSADH genes, the two plasmids pKITE301PRhSMO and pKITE103P-LSADH were constructed as follows. First, to construct a pKITE301P plasmid in which the lac promoter was replaced with the gapdh promotor from K. rhizophila DC2201, an NdeI restriction site was introduced into multicloning sites of pKITE301 using a PrimeSTAR Mutagenesis Basal Kit (Takara, Shiga, Japan) according to the manufacture's protocol to yield pKITE301nde. The promotor sequence of gapdh was amplified by PCR using $K$. rhizophila DC2201 genomic DNA as a template and introduced into the pKITE301nde vector using the NdeI and NcoI restriction sites. The obtained plasmid was designated pKITE301P. The RhSMO gene was amplified by PCR using Rhodococcus sp. ST-10 genomic DNA as a template, and the PCR product was inserted into pKITE103P using NdeI and BamHI sites. A PCR fragment of the LSADH gene from Leifsonia sp. S749 was inserted into pKITE103P (Toda et al., 2017) using the NdeI and BamHI restriction sites.

The constructed plasmids were used to transform K. rhizophila DC2201 cells, and transformants were grown on DC2201 agar medium $(0.5 \%[w / v]$ glucose, $1 \%[w / v]$ tryptone, $1 \%[w / v]$ 

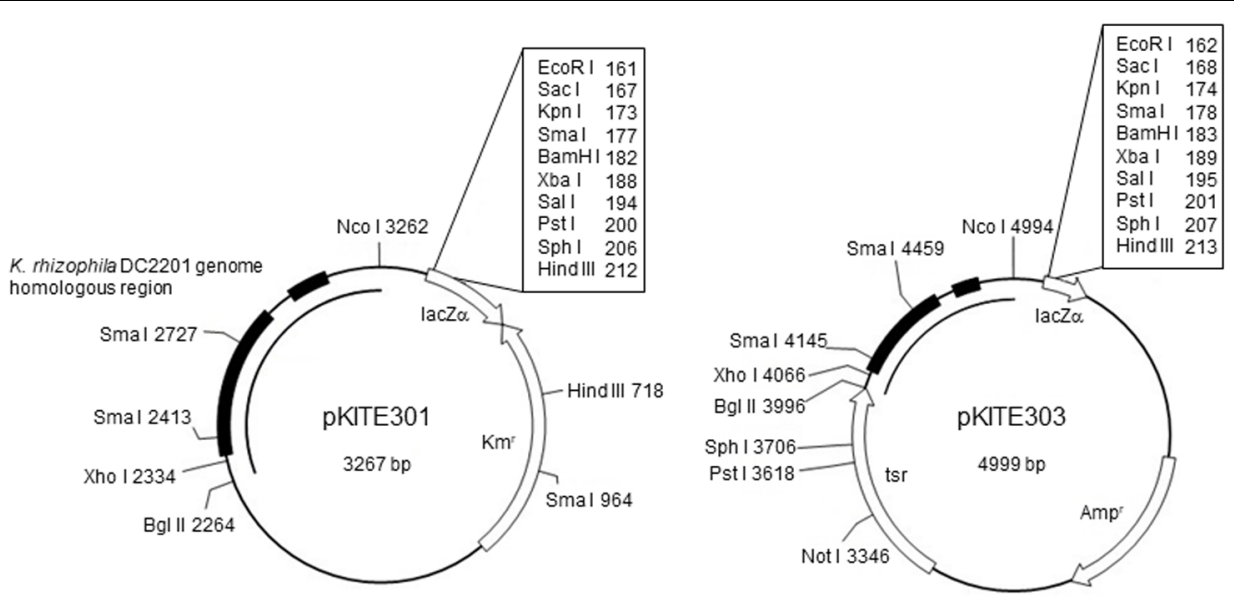

FIGURE 2 | Structure of the E. coli-Kocuria shuttle vectors derived from pKPAL3. Representative restriction endonuclease sites are shown. Solid arrows indicate the ORFs and their directions of transcription. Black boxes indicate regions of homology with the K. rhizophila DC2201 genome. Km ${ }^{r}$, kanamycin resistance gene (kanR); Ampr ${ }^{r}$, ampicillin resistance gene (bla); Thior, thiostrepton resistance gene (tsr).

yeast extract, $0.5 \%[w / v] \mathrm{NaCl}, 0.3 \%[w / v]$ bonito extract, $\mathrm{pH}$ 7.0) containing $400 \mu \mathrm{g} / \mathrm{mL}$ kanamycin and $10 \mu \mathrm{g} / \mathrm{mL}$ thiostrepton. The transformants harboring both pKITE301PRhSMO and pKITE103P-LSADH were selected and used for further experiments.

\section{Bioproduction of (S)-Styrene Oxide in a Bi-phasic System}

The K. rhizophila DC2201 cells expressing RhSMO and LSADH were grown on $50 \mathrm{~mL}$ DC2201 medium containing $400 \mu \mathrm{g} / \mathrm{mL}$ kanamycin and $10 \mu \mathrm{g} / \mathrm{mL}$ thiostrepton at $30^{\circ} \mathrm{C}$ with shaking. After cultivation, the culture medium $(50 \mathrm{~mL})$ was supplemented with $2.5 \mathrm{~mL}$ of $1 \mathrm{M}$ MOPS buffer $(\mathrm{pH} \mathrm{7.5)}$ to stabilize its $\mathrm{pH}$ and $25 \mathrm{~mL}$ of DEHP containing $400 \mathrm{mM}$ styrene and an appropriate amount of 2-propanol (5\% to $20 \%[v / v]$ in DEHP). Bioconversion was conducted at $30^{\circ} \mathrm{C}$ with shaking, and an aliquot of the organic layer $(500 \mu \mathrm{L})$ was collected after centrifugation for $5 \mathrm{~min}$. The collected samples were dried with anhydrous $\mathrm{Na}_{2} \mathrm{SO}_{4}$ and dissolved in ethyl acetate. Then, gas chromatography analysis was performed to determine the concentrations of styrene and $(S)$-styrene oxide in the reaction mixture.

\section{Gas Chromatography}

Products in the reaction mixture were analyzed using an HP 6890 GC system (Agilent Technologies, Tokyo, Japan) equipped with a Chrompack CP-cyclodextrin- $\beta$-2,3,6-M19 chiral capillary column $(0.25 \mathrm{~mm} \times 25 \mathrm{~m}, 0.25 \mu \mathrm{m}$ film; Valian, Palo Alto, CA, United States). Helium gas was used as the carrier at $15 \mathrm{psi}$, and the split ratio was 50 . Both the injector and detector temperatures were $250^{\circ} \mathrm{C}$. Measurement of the product was performed isothermally (at a column temperature of $105^{\circ} \mathrm{C}$ ) with a flame ionization detector. The retention times of styrene, $(R)$-styrene oxide, and $(S)$-styrene oxide were 2.90, 7.50, and $7.69 \mathrm{~min}$, respectively.

\section{Nucleotide Sequence Accession Numbers}

The determined nucleotide sequences of pKPAL3 from K. palustris IPUFS-1, pKITE301, pKITE303, and pKITE301P were registered with the DNA Data Bank Japan (DDBJ) under accession numbers LC317093, LC317094, LC317095, and LC317096, respectively. The nomenclatures of the constructed vectors should be standardized in the future, according to the 'Standard European Vector Architecture' database (SEVA-DB) for a coherent platform of molecular tools that are subject to a concise, and standardized format and nomenclature (Silva-Rocha et al., 2013).

\section{RESULTS}

\section{Isolation and Characterization of pKPAL3}

We isolated the novel cryptic plasmid pKPAL3 from $K$. palustris IPUFS-1 by using a transposon insertion kit in a manner similar to that described in a previous report (Toda et al., 2017). The isolated plasmid was designated pKPAL3, and its whole nucleotide sequence was determined. PKPAL3 consisted of 4,443 bp with a GC content of $63.5 \%$, and it possessed four estimated ORFs (Figure 1). BLAST searches indicated that ORF1, ORF2, and ORF3 showed approximately 40-50\% amino acid sequence identity with hypothetical proteins from other organisms, but their physiological functions remain unclear (Table 2). ORF4 exhibited an amino acid sequence identity of $48.8 \%$ with bacterial integrase/recombinase XerD from Nocardiaceae bacterium Broad-1. However, we were unable to identify genes encoding replication proteins in pKPAL3. There were two nucleotide sequence regions that showed significant identities with untranslated genomic regions of K. rhizophila DC2201 (black boxes in Figure 1). Region I consisted of 504 nucleotides and showed 76\% identity with the K. rhizophila DC2201 genome (positions 61704-62167). 
TABLE 2 | BLAST search analysis of putative ORFs of pKPAL3 from Kocuria palustris IPUFS-1.

\begin{tabular}{|c|c|c|c|c|}
\hline ORF & Position & BLAST search & Identity (\%) & Accession no. \\
\hline ORF1 & $63-743$ & Hypothetical protein from Streptococcus pneumoniae PCS70012 & 53.5 & ELU61146 \\
\hline ORF2 & $966-2003$ & Hypothetical protein from Corynebacterium epidermidicanis & 50.0 & AKK03111 \\
\hline ORF3 & $3061-3459$ & Hypothetical protein from Cellulomonas cellasea DSM 20118 & 41.7 & KGM01696 \\
\hline ORF4 & $3512-4426$ & $\begin{array}{l}\text { Putative integrase/recombinase XerD from Nocardioidaceae } \\
\text { bacterium Broad-1 }\end{array}$ & 48.8 & EGD42648 \\
\hline Region & Position & Homologous sequence (positions) & Identity (\%) & Accession no. \\
\hline Region I & $2123-2626$ & K. rhizophila DC2201, complete genome (61704 to 62167) & 76 & AP009152.1 \\
\hline \multirow[t]{10}{*}{ Region II } & $2721-2854$ & $\begin{array}{l}\text { K. rhizophila DC2201, complete genome (799495 to } 799629 ; \\
790542 \text { to } 790676 ; 1921252 \text { to } 1921379)\end{array}$ & $77-84$ & AP009152.1 \\
\hline & & $\begin{array}{l}\text { K. turfanensis strain HO-9042 genome ( } 2647231 \text { to } 2647361 \text {; } \\
3417000 \text { to } 3417130 ; 380434 \text { to } 380564 ; 1459868 \text { to } 1459991 \text {; } \\
3357701 \text { to } 3357842 ; 2547137 \text { to } 2547259)\end{array}$ & $80-81$ & CP014480.1 \\
\hline & & $\begin{array}{l}\text { K. turfanensis strain HO-9042 plasmid unnamed1 (103019 to } \\
\text { 103143; } 1885 \text { to } 2014 ; 33792 \text { to } 33917 ; 109507 \text { to } 109631 \text { ) }\end{array}$ & 79-80 & CP014481.1 \\
\hline & & K. turfanensis strain HO-9042 plasmid unnamed4 (28066 to 28195) & 79 & CP014483.1 \\
\hline & & Micrococcus luteus NCTC2665 complete genome & $77-82$ & СР001628.1 \\
\hline & & $\begin{array}{l}\text { (2439192 to } 2439324 ; 344175 \text { to } 344304 ; 2304461 \text { to } 2304592 ; \\
577570 \text { to } 577702 ; 1526931 \text { to } 1527030 ; 1558046 \text { to } 1558175 \text { ) }\end{array}$ & & \\
\hline & & $\begin{array}{l}\text { Micrococcus sp. A7 plasmid pLMA7 (65195 to } 65324 ; 79792 \text { to } \\
79924 \text { ) }\end{array}$ & $78-81$ & KJ599675.1 \\
\hline & & $\begin{array}{l}\text { Micrococcus sp. } 28 \text { plasmid pSD10 (12672 to } 12802 ; 16904 \text { to } \\
\text { 17034) }\end{array}$ & 80 & AY034092.1 \\
\hline & & Micrococcus sp. A1 plasmid pLMA1 (3973 to 4104) & 78 & LK056645.1 \\
\hline & & Micrococcus sp. V7 plasmid pLMV7 (61981 to 62111) & 79 & KF577591.1 \\
\hline
\end{tabular}

Region II consisted of 134 nucleotides, and it showed $77-84 \%$ identities with three homologous regions in the $K$. rhizophila DC2201 genome (positions 790542-790676, 799495-799629, and 1921252-1921379). Furthermore, microorganisms belonging to Micrococcaceae such as K. turfanensis and Micrococcus luteus possessed chromosomal or endogenous plasmid sequences that were homologous to portions of region II (Table 2).

\section{Minimum Sequence Region Required for Autonomous Replication in K. rhizophila DC2201}

We determined the minimum nucleotide sequence required for self-replication of pKPAL3 in K. rhizophila DC2201 through the construction of pKPAL3 deletion plasmids. Positive transformant cells were obtained that had plasmids containing both regions I and II; however, predicted ORFs encoding hypothetical proteins or the predicted recombinase (XerD) were unnecessary for autonomous replication (Figure 3).

\section{Transformation Efficiency}

As shown in Figure 3, there were significant differences in transformation efficiencies for K. rhizophila DC2201 among the various deletion plasmids. For example, pKES3310 consisted of the minimum region for replication and exhibited a transformation efficiency that was four orders of magnitude higher than that of pKES3300 possessing the entire pKPAL3 plasmid. To investigate the effect of each ORF on transformation efficiency, we constructed deletion plasmids that lacked each of the predicted ORFs and examined the transformation efficiency of host K. rhizophila DC2201 cells with each deletion plasmid (Figure 4). ORF1 and ORF2 were revealed to have a negative effect on transformation efficiency. As expected, the elimination of these ORFs from the plasmid increased the transformation efficiency of constructed plasmids by more than 100 -fold compared with that of pKES3300. On the other hand, deletion of ORF3 and/or ORF4 showed much less effect on the transformation efficiency relative to the deletion of ORF1 or ORF2.

\section{Plasmid Copy Number and Segregation Stability}

To estimate the copy number of pKITE301 in K. rhizophila DC2201, qPCR analysis was conducted on transformed $K$. rhizophila DC2201 using an approach that was similar to that described in a previous report that targeted tpi, $p g k$, and eno genes in chromosomal DNA and $k a n R$ in plasmid DNA (Toda et al., 2017). The copy number of pKITE301 was calculated to be 20 per chromosome by the ratio of the mean copy number of $k a n R$ gene to those of tpi, $p g k$, and eno.

We examined the segregation stability of pKITE301, pKES3300 and the pKES3300 deletion plasmids (pKES3300 $\Delta$ orf1 to pKES3300Aorf4; Figure 4) in K. rhizophila DC2201 transformant cells. As shown in Figure 5, pKITE301 exhibited much lower segregation stability than pKITE101, and the number of antibiotic-resistant cells harboring plasmids decreased to about 


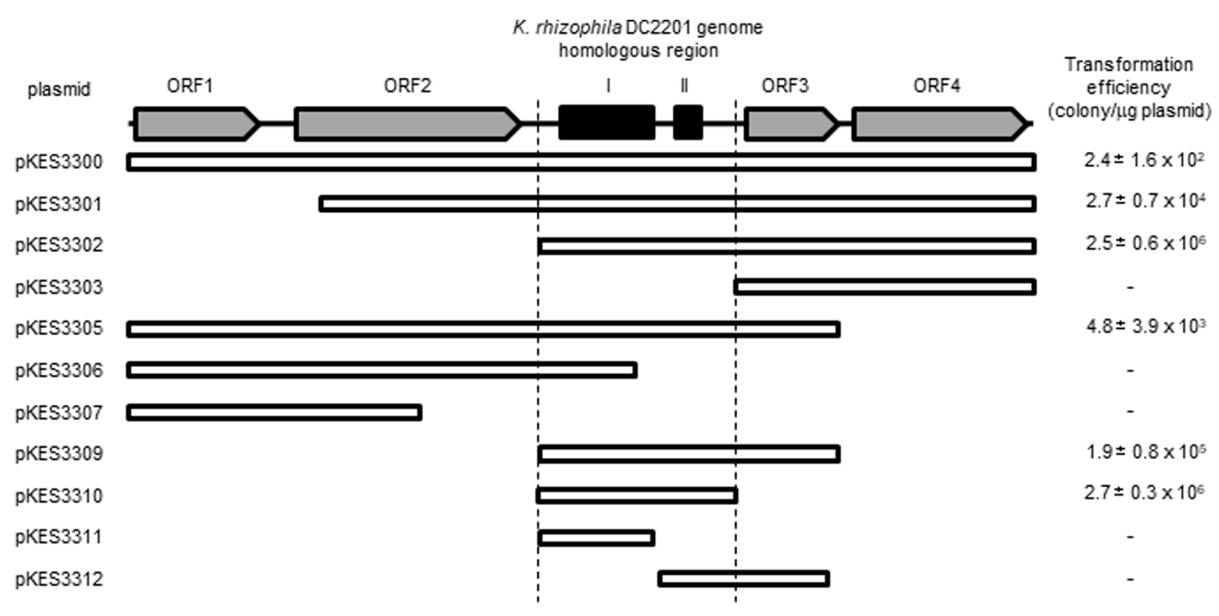

FIGURE 3 | Determination of the minimum region for self-replication of pKPAL3 derivatives in K. rhizophila DC2201 and their transformation efficiencies. Numbers indicate the transformation efficiency of the plasmids that are capable of self-replication in K. rhizophila cells.

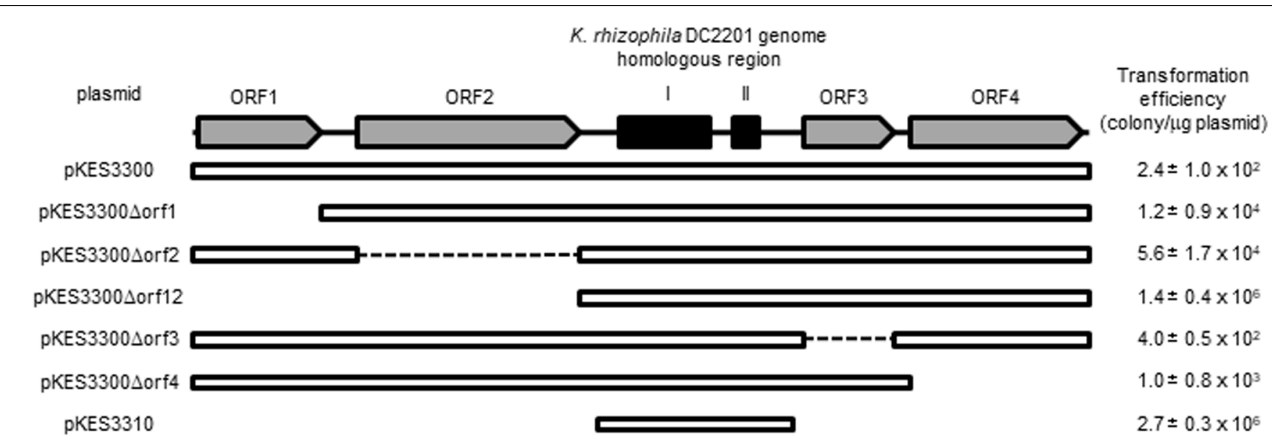

FIGURE 4 | Transformation efficiency of ORF-deleted variants derived from pKES3300. Numbers indicate the transformation efficiency of the plasmids that are capable of self-replication in K. rhizophila cells.

$2 \%$ of that of the original population after 10 subculture passages. Furthermore, a substantial decrease of segregation stability was observed only for pKES3300 $\Delta$ orf4 and pKITE101, while other deletion plasmids were stably maintained in K. rhizophila DC2201 cells (Supplementary Figure S1).

\section{Compatibility of pKITE301 and pKITE103 in Kocuria Cells}

To confirm the compatibility of the novel shuttle vector pKITE301 $\left(\mathrm{Km}^{\mathrm{r}}\right)$, the vector was transformed into K. rhizophila DC2201 cells harboring pKITE103 (Thior), and transformant cells that demonstrated resistance to both kanamycin and thiostrepton were selected. K. rhizophila cells harboring both plasmids were then confirmed by agarose gel electrophoresis analysis. Both plasmids were compatible with each other, and they were able to simultaneously replicate in $K$. rhizophila transformant cells in the presence of antibiotics. We also confirmed that pKITE301 and pKITE103 derivatives are able to coexist with the Kocuria-E. coli shuttle vector pCK-PSD1 developed by Matsumura et al. (2012) in K. rhizophila, indicating they are compatible with each other (data not shown).

\section{Transformation of Kocuria Species with pKITE301}

The self-replication of the constructed shuttle vectors was tested in other Kocuria species (Table 3). pKITE301 showed a broad range of compatibility with various Kocuria species, and the transformation efficiencies for these microorganisms were two to four orders of magnitude higher than those for pKITE101 except for K. rhizophila NBRC 16319. However, no successful transformant was observed for K. kristinae NBRC 15354 and $K$. polaris NBRC 103063. We also attempted transformation of M. luteus NBRC3333 cells using pKITE301, but no transformant was observed (data not shown).

\section{Bioproduction of (S)-Styrene Oxide Using a K. rhizophila RhSMO-LSADH Co-expression Biocatalyst}

To develop biocatalysis systems for producing enantiopure epoxides, the RhSMO-coding gene was heterologously expressed in K. rhizophila DC2201 cells under the control of a gapdh promotor using pKITE301P. LSADH, an alcohol dehydrogenase from Leifsonia sp. S749, was also expressed in K. rhizophila cells 
TABLE 3 | Transformation efficiencies of pKITE301 and pKITE101 for Kocuria species.

\begin{tabular}{|c|c|c|}
\hline Strain & 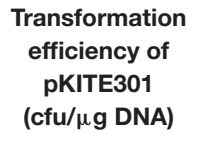 & $\begin{array}{c}\text { Transformation } \\
\text { efficiency of } \\
\text { pKITE101 } \\
(\mathrm{cfu} / \mu \mathrm{g} \text { DNA) }\end{array}$ \\
\hline K. rhizophila DC2201 & $2.7 \pm 0.3 \times 10^{6}$ & $5.3 \times 10^{2}$ \\
\hline K. kristinae NBRC 15354 & $N D^{b}$ & ND \\
\hline K. varians NBRC 15358 & $4.7 \pm 1.1 \times 10^{5}$ & $2.8 \times 10^{3}$ \\
\hline K. palustris NBRC 16318 & $1.1 \pm 0.2 \times 10^{4}$ & $1.5 \times 10^{2}$ \\
\hline K. rhizophila NBRC 16319 & $8 \pm 1.4 \times 10$ & $5 \times 10^{2}$ \\
\hline K. polaris NBRC 103063 & ND & ND \\
\hline K. flava NBRC 107626 & $2.5 \pm 1.4 \times 10^{6}$ & ND \\
\hline K. turfanensis NBRC 107627 & $3.1 \pm 0.3 \times 10^{5}$ & $1 \times 10^{2}$ \\
\hline
\end{tabular}

a Data from Toda et al. (2017).

${ }^{\mathrm{b}} \mathrm{ND}$, not detected.

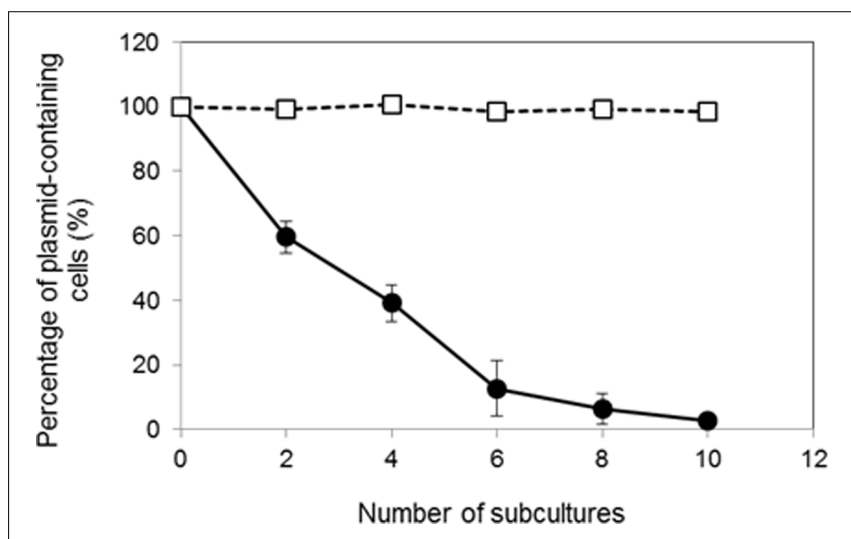

FIGURE 5 | Comparison of segregation stability of pKITE301 and pKITE101 in K. rhizophila DC2201 cells. The percentage of transformant cells possessing shuttle vectors after each subculture passage without selective antibiotics is shown: •, pKITE301; $\square$, pKITE101 (data from Toda et al., 2017). The error bars indicate standard deviations (SDs) of three measurements.

using pKITE103P for regenerating NADH by using 2-propanol as a hydrogen donor. Bioconversion of styrene to $(S)$-styrene oxide was conducted in the organic solvent-water biphasic system because the produced epoxide is unstable under aqueous conditions. Table 4 shows that the biocatalyst expressing both RhSMO and LSADH genes successfully produced $(S)$-styrene oxide in the presence of 2-propanol. Under the same conditions, a negligible amount of product was obtained by Kocuria cells possessing pKITE301P-RhSMO/pKITE103P. The control biocatalyst harboring pKITE301P/pKITE103P-LSADH showed no production of styrene oxide.

To optimize the bioconversion, the effect of 2-propanol concentration in the organic solvent was investigated. As shown in Figure 6, the product concentration generally increased in accordance with the increased 2-propanol concentration, and the highest production level of $(S)$-styrene oxide was obtained in the presence of $15 \%(v / v)$ 2-propanol in the organic solvent. Under this condition, the product concentration reached $235 \mathrm{mM}$ in a 30 -h reaction. However, styrene oxide production decreased

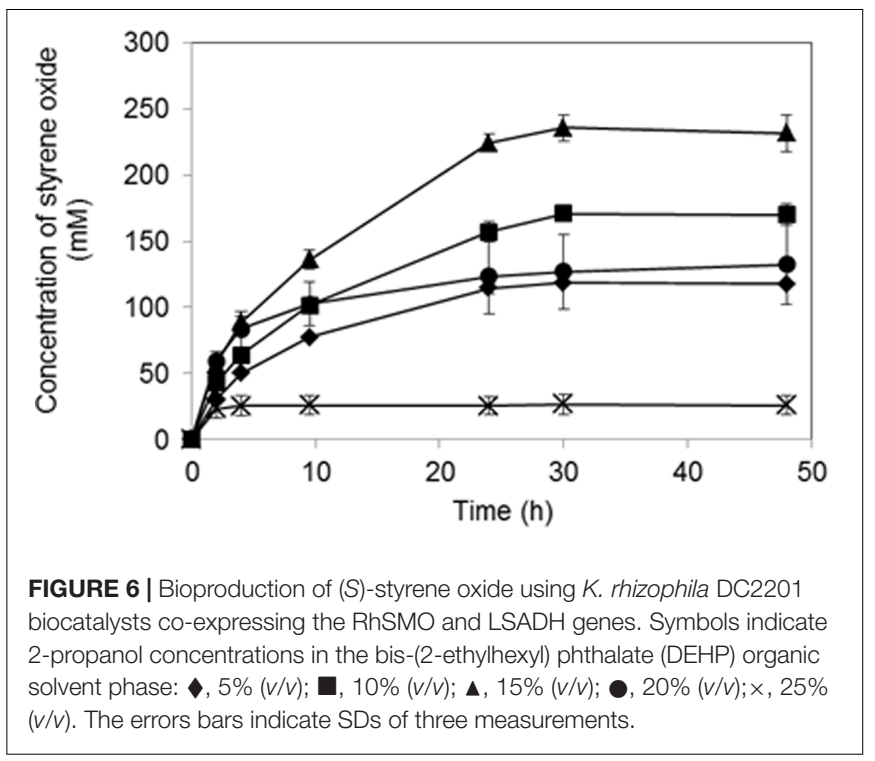

at a 2-propanol concentration of $20 \%(v / v)$, and a low yield of $(S)$-styrene oxide was obtained at a $25 \%(v / v)$ 2-propanol concentration.

\section{DISCUSSION}

Recently, we demonstrated that $K$. rhizophila DC2201 was a suitable host cell strain for producing aliphatic epoxyalkanes because they have robust cell structure and organic solvent tolerance (Toda et al., 2015). Developing genetic tools for Kocuria spp. is important for increasing the availability of this microorganism for industrial use to produce various organic compounds.

In our previous study, we isolated the two cryptic plasmids pKPAL1 and pKPAL2 from $K$. palustris IPUFS-1 using the transposon-plasmid rescue method (Toda et al., 2017). Unfortunately, we were unable to generate shuttle vectors from pKPAL2. In that study, other plasmids were detected in the extracted plasmid sample. Thus, K. palustris IPUFS-1 was assumed to have more than three cryptic plasmids in their cells. In order to construct a biocatalyst, a combination of compatible plasmid vectors expressing multiple genes in one microorganism cell is often required. In this study, we successfully identified the cryptic plasmid pKPAL3 from K. palustris IPUFS-1 and constructed a novel E. coli-Kocuria shuttle vector that is compatible with the pKITE101 plasmid previously constructed.

pKPAL3 possesses four putative ORFs, which showed high sequence identity with hypothetical proteins from Gram-positive bacteria, although the physiological roles of all ORFs remain unclear except for that of ORF4 (Table 2). The amino acids sequence of ORF4 showed high sequence identity with the putative recombinase/integrase XerD from N. bacterium Broad1. Blakely et al. (1993) reported that the XerC/D recombinase system is involved in the stable inheritance of the ColE-1related circular plasmid, and it also maintains a portion of 
TABLE 4 | Comparison of styrene oxide production by K. rhizophila DC2201 biocatalysts.

\begin{tabular}{|c|c|c|c|}
\hline Plasmid & Hydrogen source ${ }^{a}$ & Gene & Styrene oxide $(\mathrm{mM})^{\mathrm{b}}$ \\
\hline pKITE301P-RhSMO/pKITE103P & 2-propanol & RhSMO & $1.1 \pm 0.8$ \\
\hline pKITE301P-RhSMO/pKITE103P-LSADH & 2-propanol & RhSMO, LSADH & $47.0 \pm 2.7$ \\
\hline pKITE301P/pKITE103P-LSADH & 2-propanol & LSADH & 0 \\
\hline
\end{tabular}

${ }^{\mathrm{a}}$ Reactions were performed in the presence of $5 \%(\mathrm{~W} / \mathrm{v})$ 2-propanol in the organic solvent phase with $100 \mathrm{mM}$ styrene. ${ }^{\mathrm{b}}$ Product concentration in the organic phase.

chromosomal DNA from E. coli (Blakely and Sherratt, 1994). Furthermore, the resU gene, which is a member of the XerD recombinase gene family in the cryptic plasmid pUE10 from Deinococcus radiodurans, is involved in the stable replication and inheritance of pUE10 derivatives, and $\Delta$ res $U$ derivatives decreased in segregation stability in D. radiodurans cells (Meima and Lidstrom, 2000). These findings suggest that ORF4 may be involved in stabilizing segregation and inheritance of pKPAL3 in Kocuria cells. Indeed, segregation stability significantly decreased only in pKES3300 $\Delta$ orf4 compared with original pKES3300 and other ORF-deleted derivatives as shown in Supplementary Figure S1. This result supported our speculation.

Notably, pKPAL3 contained two nucleotide sequence regions (regions I and II; Figure 1) that showed significant sequence identities with genomic DNA from K. rhizophila DC2201. At least one and three conserved nucleotide sequences corresponding to regions I and II of pKPAL3 were confirmed in $K$. rhizophila DC2201 genomic DNA, respectively. These nucleotide sequences are located upstream of the IS 1380 family transposase (region I) and the IS481 family transposase (region II), and neither codes for a polypeptide. Additionally, several Kocuria and Micrococcus species, including $K$. turfanensis and $M$. luteus, have sequences that are homologous to regions I and II in their chromosomal and plasmid sequences (Table 2). Most of these sequences are located upstream of transposases as observed in K. rhizophila DC2201. These findings suggested that such nucleotide sequences have been horizontally transferred from the same origin via the transposase gene.

To determine the minimum sequence region required for autonomous replication of pKPAL3 in Kocuria cells, deletion plasmids of pKPAL3 were constructed and used to transform a series of $K$. rhizophila DC2201 cells. Figure 3 shows that no predicted ORFs are required for autonomous replication, whereas regions I and II are essential for selfreplication of pKPAL3 in Kocuria cells. Furthermore, we found that transformation efficiency varied among plasmid derivatives. Therefore, we investigated the effect of each ORF on transformation frequency using a deletion series. The results shown in Figure 4 revealed that the deletion of each ORF except ORF3 increased transformation efficiency of pKPAL3 plasmids. Particularly, the deletion of ORF1 and/or ORF2 substantially increased transformation efficiency, whereas the effect of ORF4 deletion was less than that of ORF1 or ORF2 deletion. Maier et al. (2004) also reported that a deletion derivative of an E. coli-Francisella shuttle vector lacking several ORFs exhibited higher transformation frequency than did the native plasmid. It is generally known that transformation efficiency is affected by modifications of plasmids by methylation in host cells and the configuration of plasmid DNA with respect to oligomerization and catenation (Ehrlich, 1977; Gryczan et al., 1978; Mottes et al., 1979; Canosi et al., 1981). Therefore, we deduced that differences in transformation efficiency of pKPAL3 derivatives were a consequence of the configurations or modifications caused by the deletion of ORFs in each plasmid, although the specific mechanism of this effect remains unclear.

To estimate the copy number of pKITE301 in culture, qPCR was performed using whole DNA extracted from K. rhizophila DC2201 harboring pKITE301 as a DNA template. In culture, pKITE301 showed lower copy number than pKITE101 (Toda et al., 2017), and it was calculated to be 20 per chromosome. In comparison with pKITE101, pKITE301 exhibited lower segregation stability and disappeared during culture in the absence of antibiotics (Figure 5). Several reports have indicated that theta-replicating plasmids are stably maintained in Grampositive bacteria cultures (Kiewiet et al., 1993; Zhang et al., 1994; Seegers et al., 1995; Lee and O’Sullivan, 2006), and pKITE101 is also stably maintained in Kocuria cells (Toda et al., 2017). In contrast, pKITE301 exhibited poor stability in Kocuria cells in the absence of antibiotics. These results suggest that the replicating mechanism of pKPAL3 is different from that of the highly stable theta-type plasmid. As described earlier, the low stability of pKITE301 may be restored by the addition of ORF4 into pKITE301, although further experiments are necessary to confirm this conclusion.

The transformation of various Kocuria species by the constructed shuttle vectors was tested. As shown in Table 3, several Kocuria species, including K. rhizophila NBRC 16319, $K$. varians NBRC 15358, K. palustris NBRC 16318, K. flava NBRC 107626, and K. turfanensis NBRC 107627, were successfully transformed with pKITE301 at transformation efficiencies of $8 \times 10$ to $2.5 \times 10^{6} \mathrm{cfu} / \mathrm{mg}$ DNA; however, no transformants were observed for $K$. kristinae NBRC 15354 or $K$. polaris NBRC 103063. Further optimization of conditions to prepare competent cells may be needed for these species and/or strains. Additionally, it is generally known that most bacteria have restriction modification (RM) systems that prevent the entry of foreign DNA into the host cell (Wilson and Murray, 1991). Two host cells, K. kristinae and K. polaris, are likely to have eliminated pKITE301 via their RM systems, or these strains were not competent under the general cell-transformation procedure; either of these conclusions requires confirmation. In most cases, the transformation efficiencies of pKITE301 for Kocuria species exceeded those of pKITE101, except for K. rhizophila NBRC 16319. These results indicate that the constructed shuttle vectors can be used for genetic modification of various Kocuria species. 
To confirm the utility of constructed plasmids for heterologous gene expression, we constructed the RhSMOexpression plasmid pKITE301P-RhSMO and the LSADHexpression plasmid pKITE103P-LSADH (Toda et al., 2017), and both plasmids were simultaneously transformed into K. rhizophila DC2201 cells. Under the control of the gapdh promotor from $K$. rhizophila, both RhSMO and LSADH genes were successfully expressed in transformed cells (data not shown). Using K. rhizophila cells (i.e., pKITE301P-RhSMO and pKITE103P-LSADH) as a biocatalyst, we conducted bioconversion of styrene to $(S)$-styrene oxide in an organic solvent-water biphasic reaction system. The biocatalyst harboring both RhSMO and LSADH successfully converted styrene to $(S)$-styrene oxide using 2-propanol as a hydrogen donor, whereas negligible amounts of product were obtained in the absence of 2-propanol (Table 4). Furthermore, no product was observed when a biocatalyst possessing only LSADH was used. These results indicate that both RhSMO and LSADH were successfully expressed and that LSADH functioned as a NADH regenerator in Kocuria cells. These results suggest that these shuttle vectors are more broadly applicable for constructing multi-enzymatic cascade systems for producing various compounds in Kocuria cells.

The concentration of 2-propanol (the hydrogen donor for $\mathrm{NADH}$ regeneration by $\mathrm{LSADH}$ ) in the organic solvent phase is an important factor for producing styrene oxide because it affects not only the solubility of the substrate in the aqueous phase but also the inactivation of the biocatalyst. When $15 \%(v / v)$ 2-propanol was added to the organic solvent (i.e., DEHP) phase, the highest product concentration $(235 \mathrm{mM})$ was achieved after a 30 -h reaction (Figure 6). On the other hand, styrene oxide production significantly decreased at a 2-propanol concentration

\section{REFERENCES}

Beltrametti, F., Marconi, A. M., Bestetti, G., Colombo, C., Galli, E., Ruzzi, M., et al. (1997). Sequencing and functional analysis of styrene catabolism genes from Pseudomonas fluorescens ST. Appl. Environ. Microbiol. 63, 2232-2239.

Blakely, G., May, G., McCulloch, R., Arciszewska, L. K., Burke, M., Lovett, S. T., et al. (1993). Two related recombinases are required for site-specific recombination at dif and cer in E. coli K12. Cell 75, 351-361. doi: 10.1016/00928674(93)80076-Q

Blakely, G. W., and Sherratt, D. J. (1994). Interactions of the site-specific recombinases XerC and XerD with the recombination site dif. Nucleic Acids Res. 22, 5613-5620. doi: 10.1093/nar/22.25.5613

Canosi, U., Iglesias, A., and Trautner, T. A. (1981). Plasmid transformation in Bacillus subtilis: effects of insertion of Bacillus subtilis DNA into plasmid pC194. Mol. Gen. Genet. 181, 434-440. doi: 10.1007/BF00428732

Champreda, V., Choi, Y. J., Zhou, N. Y., and Leak, D. J. (2006). Alternation of the stereo- and regioselectivity of alkene monooxygenase based on coupling protein interation. Appl. Microbiol. Biotechnol. 71, 840-847. doi: 10.1007/s00253-0050208-2

Chang, S., Galvin, J. M., and Jacobsen, E. N. (1994). Effect of chiral quaternary ammonium salts on (salen)Mn-catalyzed epoxidation of cis-olefins. a highly enantioselective, catalytic route to trans-epoxides. J. Am. Chem. Soc. 116, 6937-6938. doi: 10.1021/ja00094a059

Dufosse, L. (2006). Microbial production of food grade pigments. Food Technol. Biotechnol. 44, 313-321.

Ehrlich, S. D. (1977). Replication and expression of plasmids from Staphylococcus aureus in Bacillus subtilis. Proc. Natl. Acad. Sci. U.S.A. 74, 1680-1682. doi: 10.1073/pnas.74.4.1680 of $20 \%(v / v)$, and the lowest production level was observed for $25 \%(v / v)$ 2-propanol. Thus, K. rhizophila biocatalysts possessing the RhSMO-LSADH enzyme system exhibited the maximum epoxidation rate in the presence of 15\% $(v / v) 2$-propanol and showed resistance to $20 \%(v / v)$ 2-propanol. In corresponding E. coli recombinant biocatalyst systems, the optimum 2-propanol concentration was $6 \%(v / v)$, and the reaction was greatly inhibited at concentrations exceeding $10 \%(v / v)$, although the conditions were slightly different (Toda et al., 2012a). Thus, the K. rhizophila biocatalyst system had higher 2-propanol resistance than the corresponding E. coli system and achieved higher yields of $(S)$-styrene oxide $(235 \mathrm{mM})$ than that of the E. coli biocatalyst system $(150 \mathrm{mM})$.

We expect that the shuttle vectors developed in this study will be a powerful tool for modification of some Kocuria spp. and developing $K$. rhizophila biocatalysis systems to produce various useful water-insoluble compounds via organic synthesis.

\section{AUTHOR CONTRIBUTIONS}

HT performed the construction of the plasmid vectors from PKPAL3 and characterization of these plasmid vectors for Kocuria sp. and wrote those sections. NI planned the experimental design and wrote some parts of the manuscript.

\section{SUPPLEMENTARY MATERIAL}

The Supplementary Material for this article can be found online at: https://www.frontiersin.org/articles/10.3389/fmicb. 2017.02313/full\#supplementary-material

Farina, V., Reeves, J. T., Senanayake, C. H., and Song, J. J. (2006). Asymmetric synthesis of active pharmaceutical ingredients. Chem. Rev. 106, 2734-2793. doi: 10.1021/cr040700c

Farinas, E. T., Alcalde, M., and Arnold, F. (2004). Alkene epoxidation catalyzed by cytochrome P450 BM-3 139-3. Tetrahedron 60, 525-528. doi: 10.1016/j.tet. 2003.10.099

Goodwin, T. W. (1980). "Functions of carotenoids," in The Biochemistry of the Carotenoids, Vol. 1, ed. T. W. Goodwin (New York, NY: Chapman and Hall), 77-95. doi: 10.1007/978-94-009-5860-9_3

Gryczan, T. J., Contente, S., and Dubnau, D. (1978). Characterization of Staphylococcus aureus plasmids introduced by transformation into Bacillus subtilis. J. Bacteriol. 134, 318-329.

Hanson, R. M., and Sharpless, K. B. (1986). Procedure for the catalytic asymmetric epoxidation of allylic alcohols in the presence of molecular sieves. J. Org. Chem. 51, 1922-1925. doi: 10.1021/jo00360a058

Hartmans, S., van der Werf, M. J., and de Bont, J. A. (1990). Bacterial degradation of styrene involving a novel flavin adenine dinucleotide-dependent styrene monooxygenase. Appl. Environ. Microbiol. 56, 1347-1351.

Heipieper, H. J., Neumann, G., Cornelissen, S., and Meinhardt, F. (2007). Solvent-tolerant bacteria for biotransformations in two-phase fermentation systems. Appl. Microbiol. Biotechnol. 74, 961-973. doi: 10.1007/s00253-0060833-4

Hollmann, F., Lin, P. C., Witholt, B., and Schmid, A. (2003). Stereospecific biocatalytic epoxidation: the first example of direct regeneration of a FADdependent monooxygenase for catalysis. J. Am. Chem. Soc. 125, 8209-8217. doi: $10.1021 / \mathrm{ja0} 034119 \mathrm{u}$

Irie, R., Noda, K., Ito, Y., and Katsuki, T. (1991). Enantioselective epoxidation of unfunctionalized olefins using chiral (salen)manganese(III) 
complexes. Tetrahedron Lett. 32, 1055-1058. doi: 10.1016/S0040-4039(00) 74486-8

Katsuki, T., and Sharpless, K. B. (1980). The first paractical method for asymmetric epoxidation. J. Am. Chem. Soc. 102, 5974-5976. doi: 10.1021/ja00538 a077

Kiewiet, R., Kok, J., Seegers, J. F., Venema, G., and Bron, S. (1993). The mode of replication is a major factor in segregational plasmid instability in Lactococcus lactis. Appl. Environ. Microbiol. 59, 358-364.

Lee, J.-H., and O'Sullivan, D. J. (2006). Sequence analysis of two cryptic plasmids from Bifidobacterium longum DJO10A and construction of a shuttle cloning vector. Appl. Environ. Microbiol. 72, 527-535. doi: 10.1128/AEM.72.1.527-535. 2006

Lin, H., Qiao, J., Liu, Y., and Wu, Z. L. (2010). Styrene monooxygenase from Pseudomonas sp. LQ26 catalyzes the asymmetric epoxidation of both conjugated and unconjugated alkenes. J. Mol. Catal. B Enzym. 67, 236-241. doi: $10.1016 /$ j.molcatb.2010.08.012

Maier, T. M., Havig, A., Casey, M., Nano, F. E., Frank, D. W., and Zahrt, T. C. (2004). Construction and characterization of a highly efficient Francisella shuttle plasmid. Appl. Environ. Microbiol. 70, 7511-7519. doi: 10.1128/AEM.70. 12.7511-7519.2004

Marconi, A. M., Beltrametti, F., Bestetti, G., Solinas, F., Ruzzi, M., and Galli, E. (1996). Cloning and characterization of styrene catabolism genes from Pseudomonas fluorescens ST. Appl. Environ. Microbiol. 62, $121-127$.

Martinez, C. A., and Stewart, J. D. (2000). Cytochrome P450s potential catalysts for asymmetric olefin epoxidations. Curr. Org. Chem. 4, 263-282. doi: 10.2174/ 1385272003376265

Matsumura, E., Hamasaki, M., and Matsuyama, A. (2012). Novel expression vector. U.S. Patent No 0,142,050. Washington, DC: U.S. Patent and Trademark Office.

Meima, R., and Lidstrom, M. E. (2000). Characterization of the minimal replicon of a cryptic Deinococcus radiodurans SARK plasmid and development of versatile Escherichia coli-D. radiodurans shuttle vectors. Appl. Environ. Microbiol. 66, 3856-3867. doi: 10.1128/AEM.66.9.3856-3867. 2000

Mottes, M., Grandi, G., Sgaramella, V., Canosi, U., Morelli, G., and Trautner, T. A. (1979). Different specific activities of the monomeric and oligomeric forms of plasmid DNA in transformation of B. subtilis and E. coli. Mol. Gen. Genet. 174, 281-286. doi: 10.1007/BF00267800

Nikel, P. I., Martínez-García, E., and de Lorenzo, V. (2014). Biotechnological domestication of pseudomonads using synthetic biology. Nat. Rev. Microbiol. 12, 368-379. doi: 10.1038/nrmicro3253

Ohta, Y., Hatada, Y., Mori, K., and Nakamura, N. (2014). Plasmid vector. U.S. Patent No 8,809,048. Washington, DC: U.S. Patent and Trademark Office.

Panke, S., de Lorenzo, V., Kaiser, A., Witholt, B., and Wubbolts, M. G. (1999). Engineering of a stable whole-cell biocatalyst capable of (S)-styrene oxide formation for continuous two-liquid-phase applications. Appl. Environ. Microbiol. 65, 5619-5623.

Panke, S., Witholt, B., Schmid, A., and Wubbolts, M. G. (1998). Towards a biocatalyst for (S)-styrene oxide production: characterization of the styrene degradation pathway of Pseudomonas sp. strain VLB120. Appl. Environ. Microbiol. 64, 2032-2043.

Park, J. B, Buhler, B., Panke, S., Witholt, B., and Schmid, A. (2007). Carbon metabolism and product inhibition determine the epoxidation efficiency of solvent-tolerant Pseudomonas sp. strain VLB120DeltaC. Biotechnol. Bioeng. 98, 1219-1229. doi: 10.1002/bit.21496

Patel, R. N. (2008). Synthesis of chiral pharmaceutical intermediates by biocatalysis. Coord. Chem. Rev. 252, 659-701. doi: 10.1016/j.ccr.2007. 10.031

Porter, J. W., and Spurgeon, S. L. (eds). (1981). "Biosynthesis of carotenoids," in Biosynthesis of Isoprenoid Compounds (New York, NY: Wiley), 1-122.

Sambrook, J., and Russell, W. D. (2001). Molecular Cloning, a Laboratory Manual, 3rd Edn. Cold Spring, NY: Harbor Laboratory Press.

Seegers, J. F., Franke, C. M., Kiewiet, R., Venema, G., and Bron, S. (1995). Use of continuous culture for the selection of plasmids with improved segregational stability. Plasmid 33, 71-77. doi: 10.1006/plas.1995. 1009

Shi, Y. (2004). Organocatalytic asymmetric epoxidation of olefins by chiral ketones. Acc. Chem. Res. 37, 488-496. doi: 10.1021/ar030063x
Silva-Rocha, R., Martínez-García, E., Calles, B., Chavarría, M., Arce-Rodríguez, A., de las Heras, A., et al. (2013). The Standard European Vector Architecture (SEVA):a coherent platform for the analysis and deployment of complex prokaryotic phenotypes. Nucleic Acids Res. 41, D666-D675. doi: 10.1093/nar/ gks1119

Siriphongphaew, A., Pisnupong, P., Wongkongkatep, J., Inprakhon, P., Vangnai, A., Honda, K., et al. (2012). Development of a whole-cell biocatalyst co-expressing $\mathrm{P} 450$ monooxygenase and glucose dehydrogenase for synthesis of epoxyhexane. Appl. Microbiol. Biotechnol. 95, 357-367. doi: 10.1007/s00253-012-4039-7

Takarada, H., Sekine, M., Kosugi, H., Matsuo, Y., Fujisawa, T., Omata, S., et al. (2008). Complete genome sequence of the soil actinomycete Kocuria rhizophila. J. Bacteriol. 190, 4139-4146. doi: 10.1128/JB.01853-07

Tischler, D., Gröning, J. D., Kaschabek, S., and Schlömann, M. (2012). Onecomponent styrene monooxygenases: an evolutionary view on a rare class of flavoproteins. Appl. Biochem. Biotechnol. 167, 931-944. doi: 10.1007/s12010012-9659-y

Toda, H., Imae, R., and Itoh, N. (2012a). Efficient biocatalysis for the production of enantiopure (S)-epoxides using a styrene monooxygenase (SMO) and Leifsonia alcohol dehydrogenase (LSADH) system. Tetrahedron Asymmetry 23, 1542-1549. doi: 10.1016/j.tetasy.2012.09.017

Toda, H., Imae, R., and Itoh, N. (2014). Bioproduction of chiral epoxyalkanes using styrene monooxygenase from Rhodococcus sp. ST-10 (RhSMO). Adv. Synth. Catal. 356, 3443-3450. doi: 10.1002/adsc.201400383

Toda, H., Imae, R., Komio, T., and Itoh, N. (2012b). Expression and characterization of styrene monooxygenases of Rhodococcus sp. ST-5 and ST10 for synthesizing enantiopure (S)-epoxides. Appl. Microbiol. Biotechnol. 96, 407-418. doi: 10.1007/s00253-011-3849-3

Toda, H., and Itoh, N. (2012). Isolation and characterization of styrene metabolism genes from styrene-assimilating soil bacteria Rhodococcus sp. ST-5 and ST-10. J. Biosci. Bioeng. 113, 12-19. doi: 10.1016/j.jbiosc.2011. 08.028

Toda, H., Koyanagi, T., Enomoto, T., and Itoh, N. (2017). Characterization of two cryptic plasmids from Kocuria palustris IPUFS-1 and construction of novel Escherichia coli-Kocuria shuttle vector for biocatalysis. J. Biosci. Bioeng. 124, 255-262. doi: 10.1016/j.jbiosc.2017.03.018

Toda, H., Ohuchi, T., Imae, R., and Itoh, N. (2015). Microbial production of aliphatic (S)-epoxyalkanes by using Rhodococcus sp. strain ST-10 styrene monooxygenase expressed in organic-solvent-tolerant Kocuria rhizophila DC2201. Appl. Environ. Microbiol. 81, 1919-1925. doi: 10.1128/AEM. 03405-14

Tu, Y., Wang, Z.-X., and Shi, Y. (1996). An efficient asymmetric epoxidation method for trans-olefins mediated by a fructose-derived ketone. J. Am. Chem. Soc. 118, 9806-9807. doi: 10.1021/ja962345g

Velasco, A., Alonso, S., Garcia, J. L., Perera, J., and Diaz, E. (1998). Genetic and functional analysis of the styrene catabolic cluster of Pseudomonas sp strain Y2. J. Bacteriol. 180, 1063-1071.

Verhoef, S., Wierckx, N., Westerhof, R. G. M., de Winde, J. H., and Ruijssenaars, H. J. (2009). Bioproduction of p-hydroxystyrene from glucose by the solventtolerant bacterium Pseudomonas putida S12 in a two-phase water-decanol fermentation. Appl. Environ. Microbiol. 75, 931-936. doi: 10.1128/AEM. 02186-08

Wilson, G. G., and Murray, N. E. (1991). Restriction and modification systems. Annu. Rev. Genet. 25, 585-627. doi: 10.1146/annurev.ge.25.120191.003101

Zhang, Y., Praszkier, J., Hodgson, A., and Pittard, A. J. (1994). Molecular analysis and characterization of a broad-host-range plasmid, pEP2. J. Bacteriol. 176, 5718-5728. doi: 10.1128/jb.176.18.5718-5728.1994

Conflict of Interest Statement: The authors declare that the research was conducted in the absence of any commercial or financial relationships that could be construed as a potential conflict of interest.

Copyright $(0) 2017$ Toda and Itoh. This is an open-access article distributed under the terms of the Creative Commons Attribution License (CC BY). The use, distribution or reproduction in other forums is permitted, provided the original author(s) or licensor are credited and that the original publication in this journal is cited, in accordance with accepted academic practice. No use, distribution or reproduction is permitted which does not comply with these terms. 